\title{
Lessons Learned From an Active Shooter Full-Scale Functional Exercise In a Newly Constructed Emergency Department
}

\author{
Bryan Wexler, MD, MPH; Avram Flamm, DO, EMT-P
}

\section{ABSTRACT}

Objective: The primary objective of this exercise was to conduct a full-scale functional exercise utilizing an active-shooter-based scenario to test and evaluate hospital response and coordination with local law enforcement.

Methods: A multidisciplinary group, including community partners, formulated objectives in accordance with the Homeland Security Exercise and Evaluation Program and defined a scenario. A date to conduct the exercise was chosen on the basis of the expected completion of a large section of the new emergency department but prior to its opening for patient care.

Results: The exercise highlighted several strengths, but more importantly, illuminated areas for improvement that might otherwise have been missed in tabletop exercises and smaller-scale drills. Educational opportunities to improve functional skills and protocol were recognized.

Conclusion: Conducting a full-scale functional exercise of an active shooter in a newly constructed emergency department prior to opening for patient care provided valuable insight into areas for improvement while minimizing the impact such an exercise can have on daily operations. Should a similar opportunity arise as a result of new facilities being developed or renovations and maintenance requiring temporary closure, we advise hospitals to consider planning an exercise in the area prior to reopening for patient care. (Disaster Med Public Health Preparedness. 2017;11:522-525)

Key Words: disaster planning, mass casualty incidents, active shooter, hospital emergency preparedness

$\mathrm{T}$ he incidence and severity of mass shootings in the United States and the world has increased in the past decade. Active shooter incidents are defined as those where an individual is "actively engaged in killing or attempting to kill people in a confined and populated area." ${ }^{1}$ Active shooter incidents in the United States are becoming more common, with the incidence increasing over the past decade. ${ }^{2}$ In January 2014 the Healthcare and Public Health Sector Coordinating Council of the Federal Bureau of Investigation published guidelines for health care facilities to help mitigate this potential threat. $^{3}$ Active shooters in the health care facility setting pose a unique risk given the vulnerable population, hazardous materials, and equipment present. Within the health care facility, the emergency department (ED) is especially vulnerable to active shooters because it is a major entry portal to the hospital, allowing access to people from outside, arriving by emergency medical services and from other areas of the hospital. Targeting the ED would threaten staff, patients, and infrastructure as well as potentially delay care of casualties. In one study, it was found that ED shootings accounted for roughly one-third of health care facility shootings. ${ }^{4}$
WellSpan York Hospital is a Level 1 trauma center, serving the areas surrounding York and Adams counties in the south-central Pennsylvania region. The ED of the hospital currently sees over 80,000 visits per year, a number that has been continually increasing in volume. To help accommodate this growth, a larger ED is currently being constructed. In 2015, the WellSpan York Hospital's hazard vulnerability analysis (HVA) determined that an active shooter was its largest vulnerability. Although all hospital staff receive emergency preparedness training and protocols to respond to violence in the workplace, as well as mass casualty triage techniques during orientation and through yearly online modules, the emergency management committee felt that the lack of functional exercises in active shooter threats reduced the hospital's resilience to such an incident. To help mitigate this potential disaster, before the newly constructed ED was opened for patient care, a full-scale functional exercise was developed and executed in conjunction with community partners, utilizing an active shooter scenario. This exercise allowed for a unique opportunity of conducting a fullscale functional exercise in the ED without disrupting ongoing patient care. 


\section{METHODS}

In response to an "active shooter" event being a top priority on the hospital HVA, several months prior to the exercise, a multidisciplinary group including members from emergency management, the ED, public information, safety and security, and spiritual and mental health began formulating objectives to evaluate in accordance with the Homeland Security Exercise and Evaluation Program. The determined objectives evaluated included the following: to evaluate the ability of the ED and security staff to communicate with each other as well as with local police agencies during an armed intruder event, to test the ability of the ED and security staff to recognize and respond to an armed intruder by following the pre-defined emergency operations plan, to evaluate the ED's response to an internal mass casualty incident, and to evaluate the ability to evacuate to an alternate care site. Following the development of these objectives, in conjunction with local community responders, including law enforcement, the scenario was further defined. Approval from hospital administration and risk management departments was obtained for the scope of the exercise as well as to utilize blank cartridges for additional realism. Communication was initiated with staff to make them aware of the exercise, as were plans for signage and additional security to ensure that patients were aware of the simulation and remained safe during the entire process. A date to conduct the exercise was chosen based on the expected completion of a large section of the new ED but prior to its opening for patient care.

During the exercise, a simulated active shooter, played by a police officer from a different division than the responding local law enforcement agency, was equipped with blank cartridges and placed in the ED along with staff and simulated patients. After the exercise was initiated and the simulated perpetrator began his attack, local police departments were contacted through both 911 and panic alarms, which also notified hospital operators to place a plain-English emergency notification on hospital monitors and contact supervisors to initiate the hospital emergency operations plan. Evaluators, participants, closed circuit video, and portable video cameras provided feedback for our debriefing. All participants signed waivers for the video footage to be used for training, educational, and academic purposes.

\section{RESULTS}

The exercise highlighted several strengths, but more importantly, illuminated areas for improvement that might otherwise have been missed in tabletop exercises and smaller-scale drills. A summary of major strengths and weaknesses noted by evaluators for each objective is included in Table 1. In each objective, it was noted that staff attempted the initial steps in all objectives, but evaluators noted an obvious lack of experience in conducting a mass casualty triage. Law enforcement officers were noted to administer to the immediate threat appropriately and to interface with hospital security well, but did not communicate efficiently with ED staff to signal when an area was secure so that evacuation and treatment could commence. In addition, another interesting finding was that because the new ED was constructed to minimize noise, some staff did not hear the firearm discharge at first. After identifying multiple areas for improvement, an educational course targeting all personnel was developed and distributed. Feedback to law enforcement was also given regarding the need for improved communication with staff following an incident. These efforts will be examined further in future exercises. In addition, to aid in communication with staff, the hospital improved its mass notification capabilities with additional automated software that can communicate emergency situations by means of phone calls, text messaging, and e-mails.

\section{DISCUSSION}

With the increasing threat of violence in various forms, it is imperative that hospitals provide training to help protect staff, patients, and infrastructure. Exercises and drills can provide a means to reinforce knowledge and skill sets while identifying areas for improvement. One of the largest challenges to conducting such an intense, realistic exercise is the impact it might have on daily operations. It is noted that realistic training conditions participants toward improved response. ${ }^{5}$ As a result, the best training is often at odds with regular obligations. Conducting such training during periods when facilities are already closed to patient care, such as during construction, renovation, and maintenance periods, provides an excellent opportunity to minimize impact on operations while still achieving educational objectives. It is noted that not every institution has the opportunity to utilize renovations and new construction periods to conduct preparedness exercises. To limit impact on patient care, it is recommended to identify periods of low patient volume or to conduct drills in alternative areas to simulate the location. While these methods may not be ideal and care must be taken to examine the limitations and differences compared to a full-scale exercise in the proper location, such exercises may still be of great benefit in preparing and training staff.

Conducting a full-scale functional exercise allowed for the most realistic simulation of an active shooter in the ED. This prepared staff for an actual event far more than a tabletop exercise might have and highlighted areas for improvement that might otherwise have been missed. For example, although staff did appropriately call 911 and activate panic alarms, some staff were unfamiliar with the location and types of the new alarm systems in the recently constructed ED. Team leaders may have been educated on disaster response and triage and responded well to previous tabletop drill events, but under the pressure of a simulated active exercise, communications quickly broke down and several aberrancies in protocol were noted. While some discrepancies, such as 


\section{Major Strengths and Weaknesses Noted by Evaluators for Each Objective ${ }^{\mathrm{a}}$}

\section{Objective \\ Strengths}

Evaluate the ability of ED and security staff to

communicate with each other as well as with

local police agencies during an armed intruder

event

Test the ability of ED and security staff to

recognize and respond to an armed intruder by following

the predefined emergency operations plan

- Staff appropriately called 911 and used panic buttons to alert law enforcement

- Staff appropriately called 911 and used panic buttons to alert law enforcement

- Some clinical staff fled if possible; others hid in locked rooms if unable to flee. One staff member constructed a barricade within a patient care room; this slowed aggressor enough to allow for escape in opposite direction

- Security staff met responding police at entrance, provided assistance to police in accessing and navigating the ED

\section{Evaluate the ED's response to an internal mass} casualty incident

- Team Leader initiated role assignment

Evaluate the ability to evacuate to an alternate care site

- Staff began establishing triage areas (Red/Green/Yellow) after no immediate communication to do so

\section{Weaknesses}

- Staff on site did not effectively communicate with police, area commander, or incident commander

- Some staff were unfamiliar with the location and types of panic alarms in new ED

- Some staff hiding behind locked doors continually opened door to look for shooter

- Team leader did not communicate well, did not provide clea directions/expectations to the staff appointed to roles

- Difficult to identify Team Leader or other leadership positions during events

- Team Leader struggled with setting up disaster triage mode

- No casualty assembly point or evacuation point was identified

- No coordination of communication or care between Team Leader and attending physician

- Confusion and uncertainty among staff on the START Triage process
- Patient care was initiated where the patient was found

- Took significant prompting to get Team Leader to work through the disaster workflow

- Staff unaware of the location of the ED disaster carts or what the capabilities of these carts are 
deviating from the "run, hide, fight" or similar methodologies (staff would try to locate the active shooter by opening doors), can be attributed to the inherent artificial status of a drill no matter how realistic, these findings point to opportunities for increased education, reinforcing knowledge and skills.

In addition, while the hospital has a plan with equipment along with access set aside for law enforcement and communication between hospital security and law enforcement was excellent, additional communication between those groups and clinical staff had room for improvement. This finding is consistent with the rationale for recommendations for multidisciplinary exercises including local community partners. ${ }^{1}$

\section{CONCLUSION}

Conducting a full-scale functional exercise of an active shooter in a newly constructed ED prior to opening for patient care provided unique and valuable insight into areas for improvement in the emergency operations plan. It was a valuable opportunity to perform such an exercise without impacting patient care and allowed the local community partners to evaluate their response and communication with the hospital should such an event occur. Should a similar opportunity arise for other entities, as the result of developing new facilities or renovations and maintenance requiring temporary closure, we advise hospitals to consider planning an exercise in the area prior to reopening for patient care.

\section{About the Authors}

WellSpan, York Hospital, York, Pennsylvania (Drs Wexler and Flamm), and Drexel University College of Medicine, Philadelphia, Pennsylvania, and Penn State University College of Medicine, Hershey, Pennsylvania (Dr Wexler).

Correspondence and reprint requests to Bryan Wexler, $\mathrm{MD}$, Department of Emergency Medicine, WellSpan, York Hospital, 1001 South George Street, York, PA (e-mail: bwexler@wellspan.org).

Published online: March 6, 2017.

\section{REFERENCES}

1. Incorporating Active Shooter Planning into Health Care Facility Emergency Operations Plans. US Department of Health and Human Services. http://www.phe.gov/preparedness/planning/Documents/activeshooter-planning-eop2014.pdf. Published 2014. Accessed August 1, 2016.

2. US Department of Justice, Federal Bureau of Investigation. A study of active shooter incidents in the United States between 2000 and 2013. https://www.fbi.gov/file-repository/active-shooter-study-2000-2013-1-1. pdf/view. Accessed August 1, 2016.

3. Active shooter planning and response in a healthcare setting. Healthcare and Public Health Sector Coordinating Council, Federal Bureau of Investigation. https://www.fbi.gov/filerepository/active_shooter_planning_and_response_in_a_healthcare_setting_2 015.pdf/view. Published April 2015. Accessed August 1, 2016.

4. Kelen GD, Catlett CL, Kubit JG, et al. Hospital-based shootings in the United States: 2000 to 2011. Ann Emerg Med. 2012;60:790-798.

5. Norris WA, Wollert TN. Stress and Decision Making. Federal Law Enforcement Training Center. https://www.fletc.gov/sites/default/files/ imported_files/reference/research-papers/Stress-and-Decision-Making-0406-12-Approved-Pulic-Release-508-Accessible.pdf. Published July 11, 2011. Accessed August 1, 2016. 